\title{
Ghost dark energy model in the presence of a linear, sign-changeable interaction
}

\author{
E Ebrahimi and H Taghipour \\ Physics Faculty, Shahid Bahonar University of Kerman, Kerman, Iran \\ E-mail: ebrahimi@uk.ac.ir
}

(Received 23 October 2018 ; in final form 24 August 2019)

\begin{abstract}
In the present study,we consider the generalized ghost dark energy in the presence of a sign changeable interaction term capable of explaining the recent acceleration of the universe. We obtain different evolving parameters and plot them. We find a good agreement between the model and observations. The plots reveal that with decreasing $b$, the universe enters the acceleration phase earlier, while decreasing $\xi$ leads a delay in the entrance to the acceleration phase. Next,we present a squared sound speed analysis and find that with increasing $b$, the model can achieve the positive domain for squared sound speed, showing the signs of stability. Finally, we discuss the statefinder analysis, showing that the model can catch $\{r=1, s=0\}$ at the late time.
\end{abstract}

Keywords: cosmology, dark energy, interacting ghost dark energy, stability

For full article, refer to the Persian section 